\title{
Midazolam increases preload dependency during endotoxic shock in rabbits by affecting venous vascular tone
}

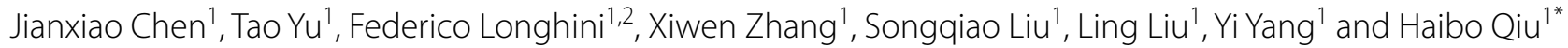

\begin{abstract}
Background: Septic patients often require sedation in intensive care unit, and midazolam is one of the most frequently used sedatives among them. But the interaction between midazolam and septic shock is not known. The aim of this study is to investigate the effects of midazolam on preload dependency in an endotoxic shock model by evaluating systemic vascular tone and cardiac function.
\end{abstract}

Methods: Eighteen rabbits were randomly divided into three groups: Control group, MID1 group and MID2 group. Rabbits underwent ketamine anaesthesia and mechanical ventilation, and haemodynamic assessments were recorded in three groups (T0). Endotoxic shock was induced by lipopolysaccharide intravenously, and fluid resuscitation and norepinephrine were administered to obtain the baseline mean arterial pressure (MAP) (T1). Rabbits received equivalent normal saline (Control) and two consecutive dosages of midazolam: $0.3 \mathrm{mg} \mathrm{kg}^{-1} \mathrm{~h}^{-1}$ (MID1) and $3 \mathrm{mg} \mathrm{kg}^{-1} \mathrm{~h}^{-1}$ (MID2) (T2). Rabbits received another round of fluid challenge and norepinephrine infusion to return the MAP to normal (T3).

Results: No significant differences in haemodynamic parameters were observed in three groups at T0, T1 or T3. Midazolam infusion significantly increased pulse pressure variation (PPV) and stroke volume variation (SVV) compared to the values in Control group, and MAP, central venous pressure (CVP), mean systemic filling pressure (Pmsf) and cardiac output (CO) decreased at T2. Same effects were observed with increasing doses of midazolam, and resistance for venous return (Rvr) decreased (MID1 vs. MID2) at T2. PPV and SVV increased significantly at T2 compared to the values at T1. MAP, CVP, Pmsf and CO decreased in MID1 and MID2 groups. Rvr also decreased in MID2 group (T2 vs. T1). Midazolam did not affect cardiac function index, systemic vascular resistance or artery resistance (T2 vs. T1).

Conclusions: Midazolam administration promoted preload dependency in septic shock models via decreased venous vascular tone without affecting cardiac function.

Keywords: Midazolam, Preload dependency, Vascular resistance, Endotoxic shock, Mean systemic filling pressure

\section{Background}

Septic shock is a deleterious systemic host response to infection characterized by hypotension that is not reversed with fluids alone. Septic shock is a common reason for admission to the intensive care unit (ICU) [1]. The response to fluid challenge is complicated by

\footnotetext{
*Correspondence: haiboq2000@163.com

1 Department of Critical Care Medicine, Zhongda Hospital, School of Medicine, Southeast University, 87 Dingjiaqiao Road, Nanjing 210009, People's Republic of China

Full list of author information is available at the end of the article
}

cardiovascular physiology, but it plays an important role in the resuscitation of sepsis patients [2]. However, fluid responsiveness only occurs in half of critically ill patients, including patients with sepsis [3]. Fluid resuscitation is a mainstay of early treatment, but the deleterious effects of excessive fluid administration that lead to tissue oedema are becoming clearer.

Patients with septic shock generally require mechanical ventilation, which makes the use of sedative drugs almost imperative to reduce anxiety and agitation and facilitate care. Benzodiazepines (e.g. midazolam) are commonly 
used to sedate patients in the ICU, and a recent survey demonstrated that midazolam remains widely used [4]. Benzodiazepines inhibit the activity of the autonomic nervous system [5, 6]. Midazolam attenuates the release of catecholamines in vivo and induces vasoplegia, which contributes to the resulting haemodynamic changes [7, 8].

Norepinephrine, an $\alpha 1$-agonist drug, is recommended as a first-line vasopressor [9]. Norepinephrine reduces the preload dependency via exertion on arterial and venous tone to increase systemic arterial resistance, primarily by recruiting blood from the large venous unstressed volume [10]. Our previous work demonstrated that propofol and dexmedetomidine increased preload dependency in an endotoxic shock model after fluid resuscitation during norepinephrine infusion, and the mechanism primarily relied on the systemic vascular system and cardiac function [11]. Few studies have reported the haemodynamic effects of midazolam infusion in endotoxic shock models during norepinephrine infusion.

In the present experimental, randomized study, we investigated the effects of midazolam on preload dependency in rabbits subjected to endotoxic shock with norepinephrine infusion by evaluating the systemic vascular system and cardiac function.

\section{Methods}

\section{Ethics statement}

New Zealand white rabbits $(3.26 \pm 0.14 \mathrm{~kg}$ body weight) were obtained from the animal centre of Southeast University and housed in a pathogen-free environment on a 12-h light/dark cycle with free food and water access for at least 5 days prior to experimentation. All animals received care according to the Helsinki convention for the use and care of animals, the "Principles of Laboratory Animal Care" formulated by the National Society for Medical Research and the "Guide for the Care and Use of Laboratory Animals" by the China National Academy of Sciences. The Academic Ethical Committee of Southeast University Medical School, Nanjing, China, approved the study protocol, which has been described previously [11].

\section{Animal preparation}

Rabbits received an intramuscular injection of ketamine $\left(20 \mathrm{mg} \mathrm{kg}^{-1}\right)$ and atropine $\left(0.15 \mathrm{mg} \mathrm{kg}^{-1}\right)$, which was used to reduce mucosal secretion in the airways. A marginal ear vein was cannulated to guarantee intravenous anaesthesia using ketamine $\left(3 \mathrm{mg} \mathrm{kg}^{-1} \mathrm{~h}^{-1}\right)$ during the entire study protocol, as previously described [11, 12]. A tracheotomy was performed after local anaesthesia with lidocaine, and a 3.5-4-mm-inner-diameter endotracheal tube was placed. Rabbits were ventilated using a Servo-I with proper software for neonatal and paediatric ventilation (Maquet Critical Care, Solna, Sweden). Tracheal cannulation was used to better adapt the rabbits to controlled mechanical ventilation and avoid spontaneous breathing. A continuous infusion of vecuronium $\left(0.05 \mathrm{mg} \mathrm{kg}^{-1} \mathrm{~h}^{-1}\right)$ was administered for neuromuscular block, and an adjunctive bolus of 0.5-1 mg was added to optimize the animal curarization if needed.

Rabbits were ventilated via volume control ventilation with the following settings: zero end-expiratory pressure, a tidal volume equal to $8 \mathrm{~mL} \mathrm{~kg}^{-1}$, an initial respiratory rate equal to 40 breath $\mathrm{min}^{-1}$ (modified according to the carbon dioxide partial pressure targeted to the physiological range) and an inspired fraction of oxygen of $60 \%$. Arterial blood was sampled for gas analysis to adjust the ventilator setting in case of respiratory acidosis prior to endotoxic shock induction. The right internal jugular vein and femoral artery were surgically isolated, and a central vein catheter was placed to infuse fluids and drugs. A dedicated arterial thermodilution catheter (4 Fr, $8 \mathrm{~cm}$ Pulsiocath PV2014L16; Pulsion Medical Systems, Munich, Germany) was inserted to acquire the haemodynamic measurements [12]. Lactate Ringer's solution $\left(4 \mathrm{~mL} \mathrm{~kg}^{-1} \mathrm{~h}^{-1}\right)$ was infused in the central vein catheter, and $2 \mathrm{~mL} \mathrm{~h}^{-1}$ of normal saline with $4 \mathrm{IU} \mathrm{mL} \mathrm{m}^{-1}$ of heparin was infused through the arterial line. Blood temperature was monitored and maintained between 38 and $39^{\circ} \mathrm{C}$ via a warming lamp.

An intravenous infusion over $30 \mathrm{~s}$ of $0.5 \mathrm{mg} \mathrm{kg}^{-1}$ E. coli LPS (O55:B5; Sigma Chem. Co., St. Louis, MO, USA) was used to induce endotoxic shock, which was confirmed by a $25 \%$ decrease in mean arterial pressure (MAP) [13]. Fluid resuscitation ( $20 \mathrm{~mL}$, intravenous bolus) was administered to all endotoxic rabbits, and $50 \mathrm{~mL} \mathrm{~kg}$ fluid was injected for another $2 \mathrm{~h}$ to maintain blood pressure. Norepinephrine infusion was initiated, and the dose was titrated to maintain MAP at baseline values and remain constant throughout the entire protocol. The haemodynamic variables were allowed to stabilize, which was assessed as a variation of MAP $<10 \%$ over a 30 -min period [14].

\section{Experimental protocol}

Rabbits were randomly divided into three groups $(n=6$ in each group): Control group, MID1 group and MID2 group. Figure 1 shows the flowchart of the study protocol. Endotoxic shock was initiated after animal preparation (T0), and the following fluid resuscitation and norepinephrine infusions were administered to all three groups. Haemodynamic measurements were obtained after stabilization (T1). Rabbits received two consecutive dosages of midazolam for $30 \mathrm{~min}: 0.3 \mathrm{mg} \mathrm{kg}^{-1} \mathrm{~h}^{-1}$ (MID1 group) and $3 \mathrm{mg} \mathrm{kg}^{-1} \mathrm{~h}^{-1}$ (MID2 group). Rabbits in the Control group received equivalent doses of normal 


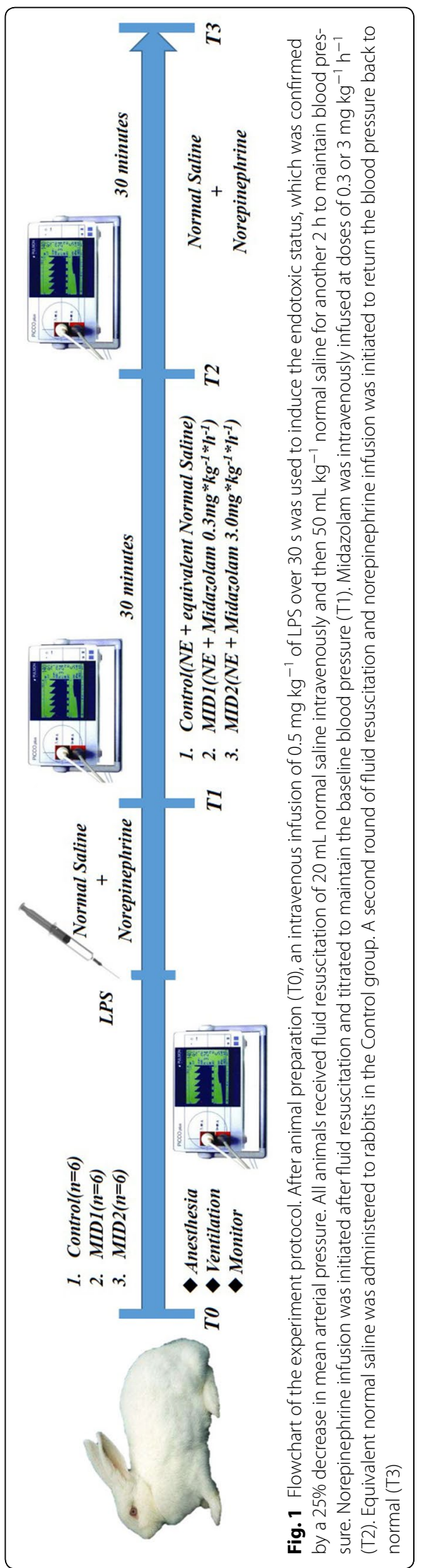


saline. Haemodynamic measurements were performed at the end of the $30 \mathrm{~min}$ trial, and the data were recorded (T2). Rabbits received another round of fluid challenge and norepinephrine infusion to return the MAP to normal (T3).

\section{Haemodynamic measurements}

Heart rate (HR), systolic blood pressure (SBP), diastolic blood pressure (DBP), MAP and central vein pressure $(\mathrm{CVP})$ were continuously monitored and recorded. Haemodynamic measurements were performed using a dedicated indwelling arterial catheter for the PiCCO Plus device (Pulsion Medical Systems, Munich, Germany).

Proper calibration of the PiCCO Plus for pulse contour analysis was performed at each measurement time point using two 3-mL bolus injections of $4{ }^{\circ} \mathrm{C}$ normal saline. A third calibrating injection was performed if the first two values differed by more than $10 \%$.

Stroke volume (SV), cardiac output (CO) and global end-diastolic volume (GEDV) were acquired via transpulmonary dilution $[11,15]$. Pulse pressure variation (PPV) and stroke volume variation (SVV) were calculated for preload dependency.

Systemic vascular resistance (Rsys), mean systemic filling pressure (Pmsf), resistance to venous return (Rvr) and arterial resistance ( $\mathrm{Ra}$ ) were calculated as previously described [14, 16]. Briefly, end-inspiratory occlusions were performed at different levels of positive end-expiratory pressure (PEEP), and the extreme values of $C O$ and CVP were recorded simultaneously. Each pair of measurements was plotted on a graph connecting $\mathrm{CO}(Y$-axis $)$ and CVP ( $X$-axis), and the regression line was computed using the least-squares method in Microsoft Excel. Pmsf was estimated as the pressure that corresponded to the $\mathrm{X}$-intercept of the regression line, and resistance to the venous return was calculated as the inverse of the slope of the line. Rsys was calculated as (MAP-CVP)/CI. Ra was estimated as (MAP-Pmsf)/CI, and Rvr was calculated as (Pmsf-CVP)/CI.

The Cardiac Function Index (CFI) was calculated as the ratio of $\mathrm{CO} \times 1000$ to GEDV, and it was recorded as an estimate of ventricular systolic function $[6,11,14,17]$. The ventilator settings, anaesthesia and vasoactive drugs were not modified during the study protocol.

\section{Blood gas measurements}

Blood gas measurements were obtained from the arterial and venous catheters at $\mathrm{T} 0, \mathrm{~T} 1, \mathrm{~T} 2$ and $\mathrm{T} 3$ to measure $\mathrm{pH}$, the partial pressure of carbon dioxide $\left(\mathrm{PCO}_{2}\right)$, the ratio of alveolar oxygen partial pressure to the fraction of inspiration $\mathrm{O}_{2}(\mathrm{P} / \mathrm{F})$, lactic acid (Lac), haemoglobin $(\mathrm{Hb})$, bicarbonate $\left(\mathrm{HCO}_{3}{ }^{-}\right)$and oxygen saturation of mixed venous blood $\left(\mathrm{SvO}_{2}\right)$.

\section{Statistics}

Data were analysed using SPSS 19.0 for Windows (SPSS Inc., Chicago, IL, USA) and GraphPad Prism 7 for Windows (GraphPad Prism Software Inc., La Jolla, CA, USA). We computed the descriptive statistics for all study variables. We used the Kolmogorov-Smirnov test and stratified the distribution plots to verify the distribution normality of the continuous variables. Data that were normally distributed are presented as the mean \pm standard deviation (SD), and non-normally distributed data are presented as medians (interquartile, IQ). We assessed differences in the distribution normality of the continuous variables using one-way analyses of variances followed by Bonferroni corrections for multiple comparisons. We used the Mann-Whitney $U$ test to evaluate non-normally distributed data. $p<0.05$ was considered statistically significant for all analyses (Table 1 ).

\section{Results}

Eighteen rabbits were anaesthetized for the study protocol. Endotoxic shock was successfully established in all animals, as indicated by a $25 \%$ decrease in MAP. Fluid resuscitation and norepinephrine infusion (Table 2) restored MAP to the initial value prior to endotoxic shock. The rabbits received a second fluid challenge and norepinephrine infusion after midazolam infusion to return the MAP to normal. No differences were detected between the Control, MID1 and MID2 groups with respect to the time to achieve endotoxic shock $(29.1 \pm 6.8$, $28.4 \pm 7.2$ and $29.0 \pm 7.0 \mathrm{~min}$, respectively; $p>0.05$ ) or the volume of administered fluid during T0-T1 and T1T2. The volume of administered fluid increased from T2 to T3 between the Control, MID1 and MID2 groups $(29.10 \pm 1.46,45.40 \pm 1.19$ and $65.21 \pm 1.16 \mathrm{~mL}$, respectively, $p<0.05)$. No differences were detected between the Control, MID1 and MID2 groups with respect to the norepinephrine infusion rate $(5.51 \pm 0.23,5.55 \pm 0.21$ and $5.56 \pm 0.27 \mathrm{mcg} \mathrm{kg}^{-1} \mathrm{~min}^{-1}$, respectively, $p>0.05$ ). Blood gases confirmed normal baseline status, and there were no significant differences between T0, T1, T2 or T3 among all three groups (Table 3). No rabbits died.

Table 1 shows the effects of midazolam on haemodynamics. No differences between the Control, MID1 and MID2 groups were observed at T0, which demonstrates that the study population was homogeneous prior to the initiation of the sedative infusion $(p>0.05)$.

\section{Effects of midazolam on preload dependency}

Table 1 shows that no differences in PPV or SVV were observed between groups at T0. No differences in PPV or SVV were observed after modelling and resuscitation between the three groups, which demonstrates that all rabbits were without fluid responsiveness at $\mathrm{T} 1$. 


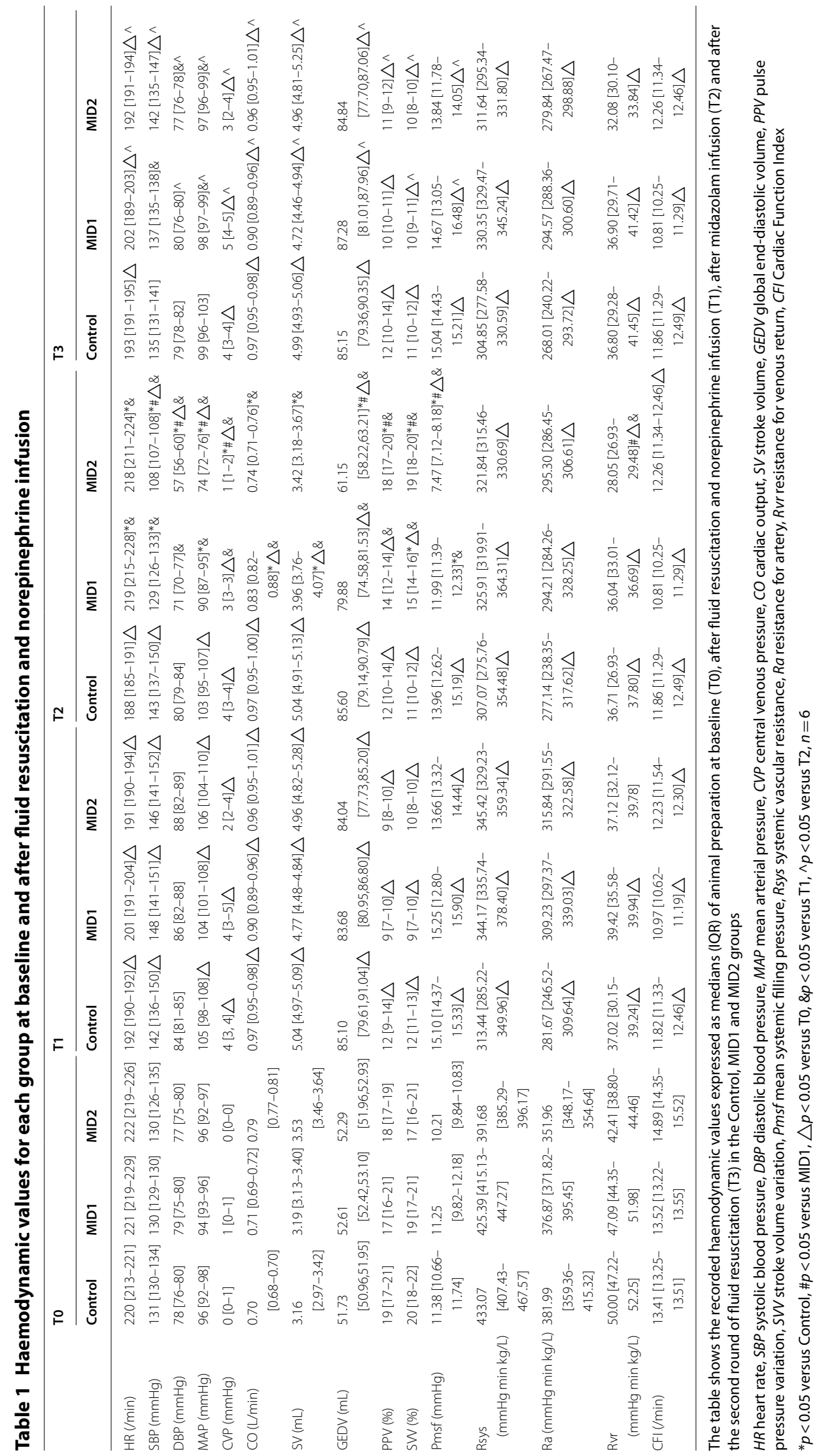


Table 2 Fluid and norepinephrine administration during the experiment

\begin{tabular}{|c|c|c|c|c|c|c|c|c|c|}
\hline \multirow[t]{2}{*}{ Treatment } & \multicolumn{3}{|l|}{ T0-T1 } & \multicolumn{3}{|l|}{$\mathrm{T} 1-\mathrm{T} 2$} & \multicolumn{3}{|l|}{ T2-T3 } \\
\hline & Control & MID1 & MID2 & Control & MID1 & MID2 & Control & MID1 & MID2 \\
\hline Saline $(m L)$ & $343.33 \pm 16.32$ & $350.00 \pm 12.64$ & $345.00 \pm 13.78$ & $29.10 \pm 1.46$ & $29.70 \pm 1.13$ & $29.25 \pm 1.24$ & $29.10 \pm 1.46$ & $45.40 \pm 1.19^{*}$ & $65.21 \pm 1.16^{*} \#$ \\
\hline $\begin{array}{l}\text { Norepinephrine } \\
\qquad\left(\mathrm{mcg} \mathrm{kg}^{-1} \mathrm{~min}^{-1}\right)\end{array}$ & $5.51 \pm 0.23$ & $5.55 \pm 0.21$ & $5.56 \pm 0.27$ & $5.51 \pm 0.23$ & $5.55 \pm 0.21$ & $5.56 \pm 0.27$ & $5.51 \pm 0.23$ & $5.55 \pm 0.21$ & $5.56 \pm 0.27$ \\
\hline
\end{tabular}

Data are shown as the mean \pm SD

T0: baseline; T1: endotoxic shock after fluid resuscitation and norepinephrine infusion; T2: after the administration of midazolam at $0.3 \mathrm{mg} \mathrm{kg}^{-1} \mathrm{~h}^{-1}$ (MID1) or $3 \mathrm{mg} \mathrm{kg}^{-1} \mathrm{~h}^{-1}$ (MID2); T3: after second round of fluid resuscitation

${ }^{*} p<0.05$ versus control, $\# p<0.05$ versus MID1

Midazolam administration significantly increased PPV in the MID2 group at T2 $(p<0.05)$, and it significantly increased SVV in the MID1 and MID2 groups $(p<0.05)$ compared to that in the Control group (Fig. 2). SVV in the MID2 group was significantly higher than that in the MID1 group at T2 $(p<0.05)$ (Fig. 1b). No differences were detected in PPV or SVV between groups at T3 $(p>0.05)$.

PPV and SVV decreased from T0 to T1 in all groups $(p<0.05)$ but increased significantly in the MID1 and MID2 groups at T2 compared to the values at T1 $(p<0.05)$. PPV and SVV decreased in the MID1 and MID2 groups from T2 to T3 $(p<0.05)$, but no differences were detected in the Control group (Table 1 and Fig. 2).

\section{Effects of midazolam on haemodynamic parameters}

As shown in Table 1, there were no significant differences in the haemodynamic parameters among the three groups at T0, T1 and T3. However, MAP and Pmsf decreased significantly in the MID1 and MID2 groups $(p<0.05)$, and CVP and CO decreased in the MID2 group compared to the values in the Control group at T2 $(p<0.05)$ (Table 1). MAP, CVP and Pmsf in the MID2 group were significantly lower than the values in the MID1 group at T2 $(p<0.05)$ (Table 1$)$.

Midazolam dosage did not affect Rsys or Ra at T2 or T3, but Rvr deceased significantly in the MID2 group compared to that in the Control group at T2 and T3 $(p<0.05)$ (Fig. 3). There were no differences in CFI between groups at T2 or T3 $(p>0.05)$.

MAP, CVP, Pmsf, CO and SV increased from T0 to $\mathrm{T} 1$ and $\mathrm{T} 2$ to T3 in all three groups $(p<0.05)$. Rsys, Ra, Rvr, HR and CFI decreased significantly from T0 to T1 $(p<0.05)$ (Table 1). MAP, CVP, Pmsf and CO decreased in the MID1 and MID2 groups at T2 compared to the values at $\mathrm{T} 1(p<0.05)$, and the opposite results occurred at T3 compared to the values at T2 (Table 1 ). Rvr only decreased in the MID2 group at T2 $(p<0.05)$. No differences were detected in Rvr from T2 to T3 (Fig. 3, $p>0.05$ ).

\section{Discussion}

To our knowledge, this study is the first to investigate the effects of two midazolam doses on haemodynamics in an endotoxic shock model during norepinephrine infusion. The main results can be summarized as follows: (1) midazolam increased the preload dependency, reduced Pmsf, CVP, GEDI and Pvr and affected the SV and CO despite the increase in HR; (2) no effects on cardiac contractile function as expressed by the CFI were observed. Thus, midazolam primarily affects the heart by increasing venous capacitance.

To better elucidate the mechanism, the venous return curve of one representative rabbit was constructed from the average values obtained for right atrial pressure (a surrogate for central venous pressure) and cardiac output (Fig. 4), as previously described [18]. Three points in Fig. 4 represent the circulatory working points at $\mathrm{T} 1(\mathrm{a})$, T2(c) and T3(d). The cardiac function curve did not change with increasing midazolam infusion rates ( $\mathrm{T} 1$ and $\mathrm{T} 2$ ), but the working point left-shifted to lower values of $\mathrm{CO}$ and right atrial pressure. The Pmsf obtained from the venous return curve was also reduced. This Pmsf reduction may be explained by an increased vascular capacitance due to midazolam infusion, which shifted the stressed volume to the unstressed volume [19]. Vascular capacity is defined as the volume at a given pressure [19], assuming that the total intravascular volume in rabbits did not change. The recorded Pmsf reduction suggests an increase in vascular capacitance. Endotoxic rabbits with midazolam-induced haemodynamic changes were resuscitated at T3 until MAP was restored to baseline (i.e. before sedative use) to further test our hypothesis. Figure 3 shows that the $C$ point returned to the $\mathrm{D}$ point, i.e. from the ascending curve to plateau status, after fluid infusion.

Augmented vascular capacitance and lower Pmsf reduced the venous return and therefore the SV and $\mathrm{CO}$, despite attempts at compensation by increasing the HR. The CFI was not affected. The preload (i.e. GEDV) 


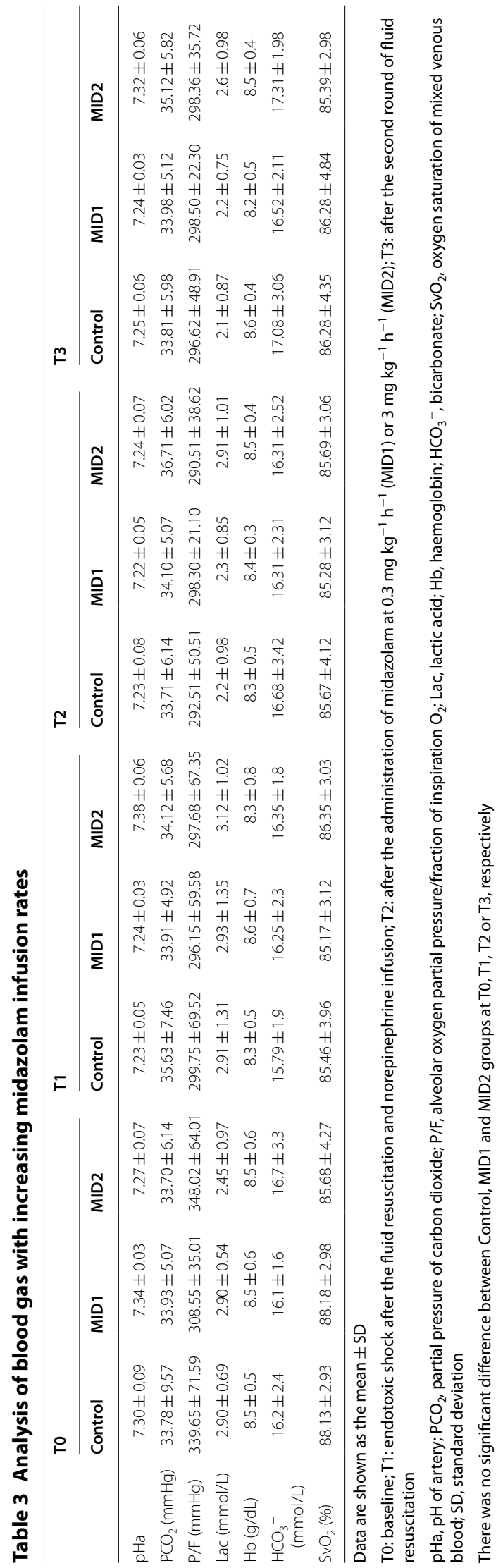



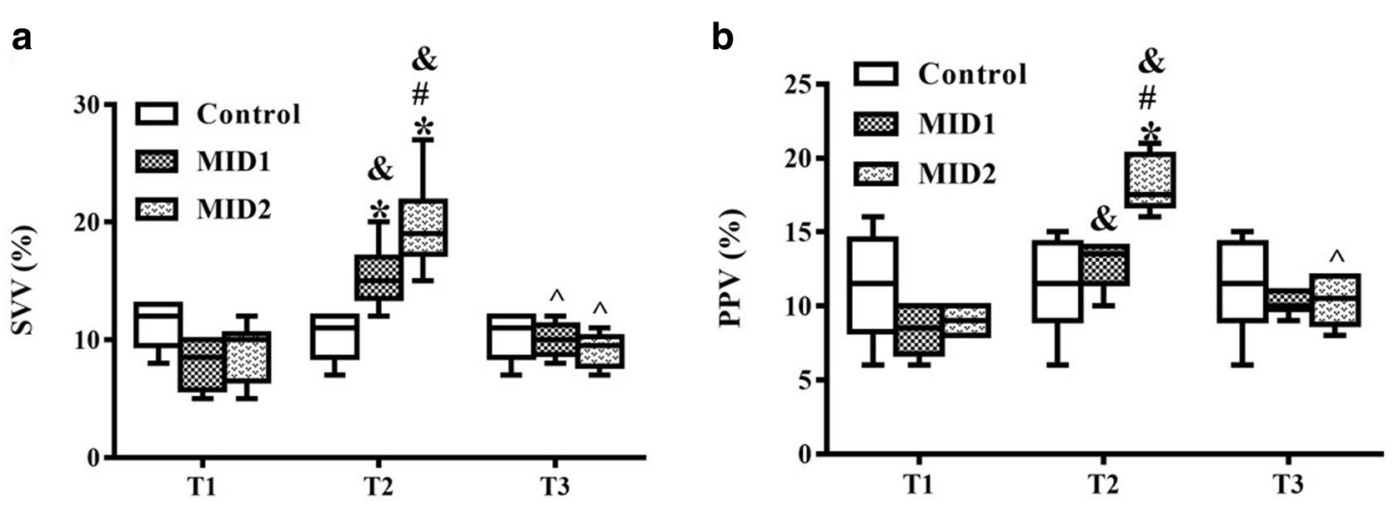

Fig. 2 Midazolam increased preload dependency of endotoxic shock rabbits. a The effects of midazolam on pulse pressure variation between the Control, MID1 and MID2 groups at T1, T2 and T3. b The effects of midazolam on stroke volume variation between the Control, MID1 and MID2 groups at T1,T2 and T3. PPV pulse pressure variation, SW stroke volume variation; ${ }^{*} p<0.05$ versus Control, $\# p<0.05$ versus MID1, \& $p<0.05$ versus T1, $\wedge p<0.05$ versus $T 2, n=6$

decreased significantly because of the reduced venous return, and the preload dependency (PPV) increased significantly. The Rvr decreased significantly from T0 to T2, which confirmed midazolam-induced venous dilatation and resulted in reduced preload and increased preload dependency.

Our study demonstrates that midazolam increases preload dependency in an endotoxic shock rabbit model. This result is inconsistent with a prior clinical observational study also conducted by our work team in which midazolam use did not increase the preload dependency in septic shock patients [20]. The following reasons may explain this inconsistency. First, the midazolam dose regimen in the prior study was a bolus dose of $2.5 \mathrm{mg}$ and continuous infusion of $1.5 \mathrm{mg} \mathrm{h}$, which is equivalent to the dose in the MID1 group in our study, and the effects on vascular tone were not obvious. Second, the sedation was titrated to Ramsay 3-4 points in the prior study, and the rabbits were anaesthetized using ketamine with midazolam. These sedatives are likely not comparable.

We recorded no differences in cardiac function as expressed by the CFI, i.e. the ratio of cardiac output to global end-diastolic volume. CFI correlates with left ventricular global systolic function $[21,22]$, and the recorded differences in SVI and CI cannot be attributable to an effect of acidosis on contractility, or on contractility itself, but to a preload midazolam effect.

Some limitations of the present study must be mentioned. First, we used SVV and PVV to reflect volume responsiveness. Previous studies demonstrated that SVV (directly measured using different pulse contour techniques or Doppler ultrasounds) or PPV reliably predicts the response to fluids when several prerequisites are met (e.g. absence of arrhythmias, tidal volume larger than $8 \mathrm{~mL} / \mathrm{kg}$, no respiratory movements) [23, 24]. These requirements were satisfied in the present study, and the use of SVV and PVV was likely reliable and effective.

Second, we used the end-inspiratory occlusion technique to draw the venous return curve for Pmsf computation [16]. Persichini et al. [14] recorded CO and CVP during end-inspiratory and end-expiratory ventilatory occlusions to describe a more precise curve. The description of this method was published after our study began, and our methods were chosen based on previously described literature.

In conclusion, midazolam affected the preload dependency at increasing doses in endotoxic shock rabbits undergoing norepinephrine infusion without affecting heart contractile function. These results suggest no major effects of midazolam on cardiac function in septic shock and that the haemodynamic fluctuations at large doses of midazolam were due to venous dilation. These data were derived from animal models, and further studies must be performed in humans to understand the possible interference of benzodiazepine in septic shocked patients.

\section{Conclusions}

In conclusion, a high dose of midazolam administration in a septic shock model after fluid resuscitation and norepinephrine infusion increased the preload dependency via modification of vascular resistance. No effects on cardiac function were observed. Further studies must be performed in humans to understand the possible interference of sedative drugs on haemodynamics during septic shock. 

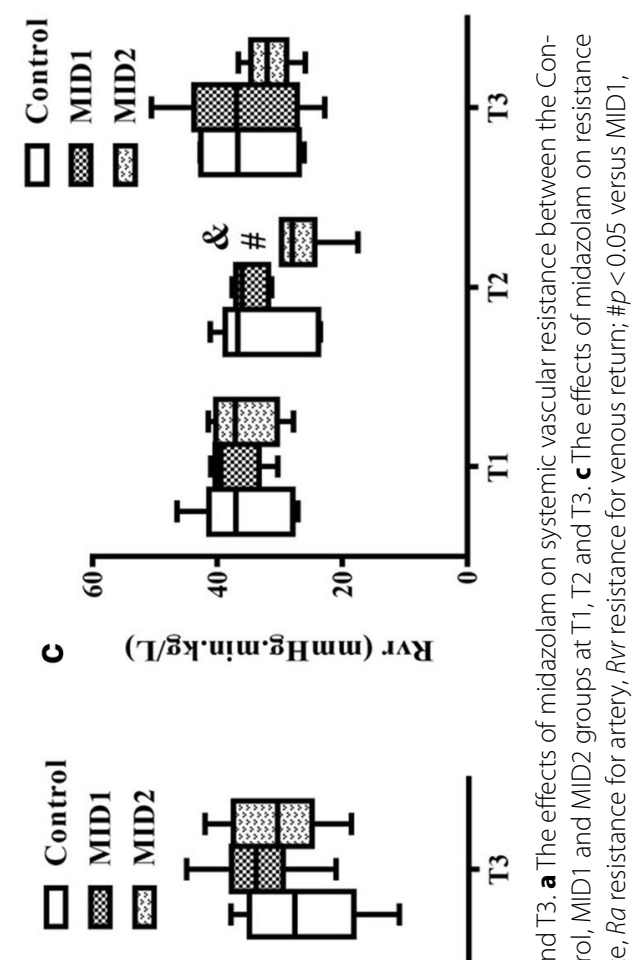

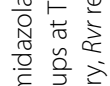

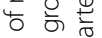

苾

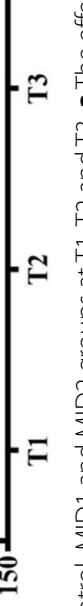

兽

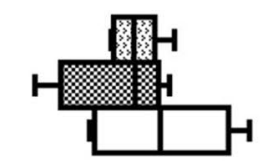

它 它

宁垔

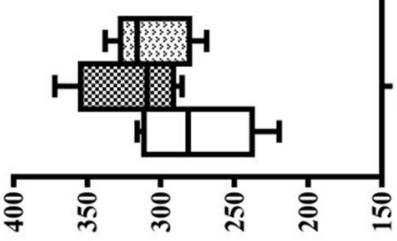

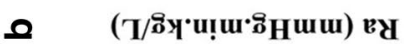

E

ปิ

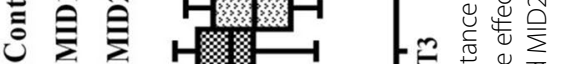

口罒
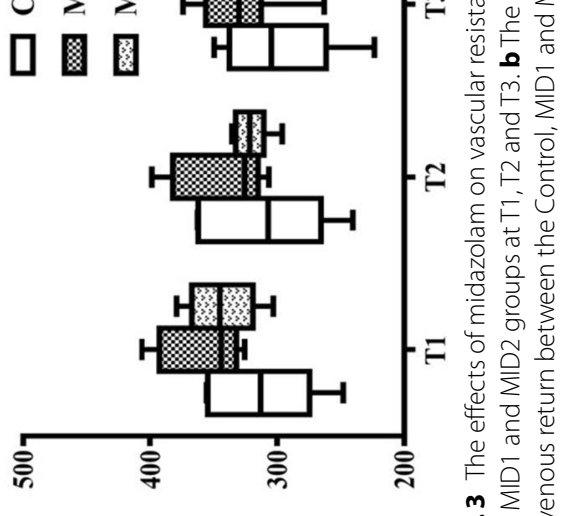

远

$\pi$

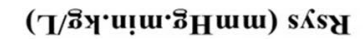




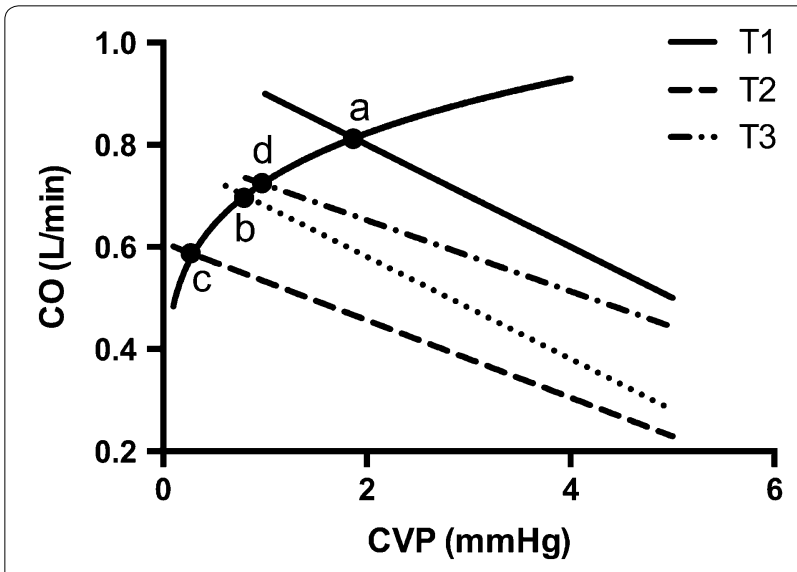

Fig. 4 Schematic diagram of the effects of midazolam. Venous return curve and cardiac output curve constructed from the average values of central venous pressure, mean systemic filling pressure and cardiac output after resuscitation and midazolam infusion. The dots are the values derived from Table 1. (a) The working point of the circulation during $\mathrm{T} 1$; (b) the volume effect of generalized vasodilatation on $\mathrm{CO}$ by midazolam; (c) an additional effect of midazolam on resistance for venous return; (d) the volume effect of generalized vasodilatation on CO by fluid administration after midazolam. Cardiac output; CVP central venous pressure

\section{Authors' contributions}

JX.C participated in acquisition, analysis, interpretation of data and contributed to the manuscript writing. T.Y participated in the execution of the study, data collection and analysis. F.L and XW.Z conducted the data analysis and participated in manuscript writing. SQ.L, L.L, Y.Y and HB.Q participated in the study design and reviewed the intellectual content. HB.Q participated in study design and coordination of all the study. All authors read and approved the final manuscript.

\section{Author details}

1 Department of Critical Care Medicine, Zhongda Hospital, School of Medicine, Southeast University, 87 Dingjiaqiao Road, Nanjing 210009, People's Republic of China. ${ }^{2}$ Anesthesia and Intensive Care, Department of Translational Medicine, Eastern Piedmont University "A. Avogadro", Novara, Italy.

\section{Acknowledgements}

Assistance with the study: there was no assistance with the study.

\section{Competing of interests}

The authors declare that they have no competing of interests.

\section{Ethics approval and consent to participate}

All animals received care according to the Helsinki convention for the use and care of animals, the "Principles of Laboratory Animal Care" formulated by the National Society for Medical Research and the "Guide for the Care and Use of Laboratory Animals" by the China National Academy of Sciences. The Academic Ethical Committee of Southeast University Medical School, Nanjing, China, approved the study protocol.

\section{Funding}

National Natural Science Foundations of China (81501705); Projects of Jiangsu province's medical key discipline (889-KJXW11.3); Special Fund for Health-Scientific Research in the Public Interest Program (No. 201202011); Postgraduate Research and Practice Innovation Program of Jiangsu Province (KYCX17_0168).

\section{Publisher's Note}

Springer Nature remains neutral with regard to jurisdictional claims in published maps and institutional affiliations.

Received: 27 November 2017 Accepted: 23 April 2018

Published online: 02 May 2018

\section{References}

1. Angus DC, van der Poll T. Severe sepsis and septic shock. N Engl J Med. 2013:369:2063

2. Monnet X, Marik PE, Teboul JL. Prediction of fluid responsiveness: an update. Ann Intensive Care. 2016;6:111.

3. Michard F, Teboul JL. Predicting fluid responsiveness in ICU patients: a critical analysis of the evidence. Chest. 2002;121:2000-8.

4. Barr J, Fraser GL, Puntillo K, Ely EW, Gelinas C, Dasta JF, Davidson JE, Devlin JW, Kress JP, Joffe AM, Coursin DB, Herr DL, Tung A, Robinson BR, Fontaine DK, Ramsay MA, Riker RR, Sessler CN, Pun B, Skrobik Y, Jaeschke R, American College of Critical Care M. Clinical practice guidelines for the management of pain, agitation, and delirium in adult patients in the intensive care unit. Crit Care Med. 2013;41:263-306.

5. Fujiwara $Y$, Ito H, Asakura Y, Sato Y, Nishiwaki K, Komatsu T. Preoperative ultra short-term entropy predicts arterial blood pressure fluctuation during the induction of anesthesia. Anesth Analg. 2007;104:853-6.

6. Royse CF, Liew DF, Wright CE, Royse AG, Angus JA. Persistent depression of contractility and vasodilation with propofol but not with sevoflurane or desflurane in rabbits. Anesthesiology. 2008;108:87-93.

7. Ledowski T, Bein B, Hanss R, Paris A, Fudickar W, Scholz J, Tonner PH. Neuroendocrine stress response and heart rate variability: a comparison of total intravenous versus balanced anesthesia. Anesth Analg. 2005:101:1700-5.

8. Rutledge C, Brown B, Benner K, Prabhakaran P, Hayes L. A novel use of methylene blue in the pediatric ICU. Pediatrics. 2015;136:e1030-4.

9. Dellinger RP, Levy MM, Rhodes A, Annane D, Gerlach H, Opal SM, Sevransky JE, Sprung CL, Douglas IS, Jaeschke R, Osborn TM, Nunnally ME, Townsend SR, Reinhart K, Kleinpell RM, Angus DC, Deutschman CS, Machado FR, Rubenfeld GD, Webb SA, Beale RJ, Vincent JL, Moreno R, Surviving Sepsis Campaign Guidelines Committee including the Pediatric S. Surviving sepsis campaign: international guidelines for management of severe sepsis and septic shock: 2012. Crit Care Med. 2013;41:580-637.

10. Monnet X, Jabot J, Maizel J, Richard C, Teboul JL. Norepinephrine increases cardiac preload and reduces preload dependency assessed by passive leg raising in septic shock patients. Crit Care Med. 2011:39:689-94.

11. Yu T, Li Q, Liu L, Guo F, Longhini F, Yang Y, Qiu H. Different effects of propofol and dexmedetomidine on preload dependency in endotoxemic shock with norepinephrine infusion. J Surg Res. 2015;198:185-91.

12. Liet JM, Jacqueline C, Orsonneau JL, Gras-Leguen C, Potel G, Roze JC. The effects of milrinone on hemodynamics in an experimental septic shock model. Pediatr Crit Care Med J Soc Crit Care Med World Fed Pediatr Intensive Crit Care Soc. 2005;6:195-9.

13. Wiel E, Pu Q, Leclerc J, Corseaux D, Bordet R, Lund N, Jude B, Vallet B. Effects of the angiotensin-converting enzyme inhibitor perindopril on endothelial injury and hemostasis in rabbit endotoxic shock. Intensive Care Med. 2004;30:1652-9.

14. Persichini R, Silva S, Teboul JL, Jozwiak M, Chemla D, Richard C, Monnet X. Effects of norepinephrine on mean systemic pressure and venous return in human septic shock. Crit Care Med. 2012;40:3146-53.

15. Freitas FG, Bafi AT, Nascente AP, Assuncao M, Mazza B, Azevedo LC, Machado FR. Predictive value of pulse pressure variation for fluid responsiveness in septic patients using lung-protective ventilation strategies. $\mathrm{Br}$ J Anaesth. 2013;110:402-8.

16. Maas JJ, Geerts BF, van den Berg PC, Pinsky MR, Jansen JR. Assessment of venous return curve and mean systemic filling pressure in postoperative cardiac surgery patients. Crit Care Med. 2009;37:912-8. 
17. Schmidt C, Roosens C, Struys M, Deryck YL, Van Nooten G, Colardyn F, Van Aken $\mathrm{H}$, Poelaert Jl. Contractility in humans after coronary artery surgery. Anesthesiology. 1999;91:58-70.

18. Maas JJ, Pinsky MR, de Wilde RB, de Jonge E, Jansen JR. Cardiac output response to norepinephrine in postoperative cardiac surgery patients: interpretation with venous return and cardiac function curves. Crit Care Med. 2013;41:143-50.

19. Gelman S. Venous function and central venous pressure: a physiologic story. Anesthesiology. 2008;108:735-48.

20. Yu T, Peng X, Liu L, Li Q, Huang Y, Guo F, Yang Y, Qiu H. Propofol increases preload dependency in septic shock patients. J Surg Res. 2015;193:849-55.

21. Jabot J, Monnet X, Bouchra L, Chemla D, Richard C, Teboul JL. Cardiac function index provided by transpulmonary thermodilution behaves as an indicator of left ventricular systolic function. Crit Care Med. 2009;37:2913-8.
22. Ritter S, Rudiger A, Maggiorini M. Transpulmonary thermodilutionderived cardiac function index identifies cardiac dysfunction in acute heart failure and septic patients: an observational study. Crit Care. 2009;13:R133.

23. De Backer D, Heenen S, Piagnerelli M, Koch M, Vincent JL. Pulse pressure variations to predict fluid responsiveness: influence of tidal volume. Intensive Care Med. 2005;31:517-23.

24. De Backer D, Taccone FS, Holsten R, Ibrahimi F, Vincent JL. Influence of respiratory rate on stroke volume variation in mechanically ventilated patients. Anesthesiology. 2009;110:1092-7.

\section{Submit your manuscript to a SpringerOpen ${ }^{\circ}$ journal and benefit from:}

- Convenient online submission

- Rigorous peer review

- Open access: articles freely available online

- High visibility within the field

- Retaining the copyright to your article

Submit your next manuscript at $\boldsymbol{\nabla}$ springeropen.com 